\title{
Acute renal metabolic effect of metformin assessed with hyperpolarised MRI in rats
}

\author{
Haiyun $\mathrm{Qi}^{1}$ • Per M. Nielsen ${ }^{1}$ • Marie Schroeder ${ }^{1}$ - Lotte B. Bertelsen ${ }^{1}$. Fredrik Palm ${ }^{2}$. \\ Christoffer Laustsen ${ }^{1}$ (D)
}

Received: 10 April 2017 / Accepted: 4 August 2017 / Published online: 21 September 2017

(C) Springer-Verlag GmbH Germany 2017

\begin{abstract}
Aims/hypothesis Metformin inhibits hepatic mitochondrial glycerol phosphate dehydrogenase, thereby increasing cytosolic lactate and suppressing gluconeogenesis flux in the liver. This inhibition alters cytosolic and mitochondrial reductionoxidation (redox) potential, which has been reported to protect organ function in several disease states including diabetes. In this study, we investigated the acute metabolic and functional changes induced by metformin in the kidneys of both healthy and insulinopenic Wistar rats used as a model of diabetes. Methods Diabetes was induced by intravenous injection of streptozotocin, and kidney metabolism in healthy and diabetic animals was investigated 4 weeks thereafter using hyperpolarised ${ }^{13} \mathrm{C}$-MRI, Clark-type electrodes and biochemical analysis.

Results Metformin increased renal blood flow, but did not change total kidney oxygen consumption. In healthy rat kidneys, metformin increased $\left[1-{ }^{13} \mathrm{C}\right]$ lactate production and reduced mitochondrial $\left[1{ }^{13} \mathrm{C}\right]$ pyruvate oxidation (decreased the ${ }^{13} \mathrm{C}$-bicarbonate/ $\left[1-{ }^{13} \mathrm{C}\right]$ pyruvate ratio) within $30 \mathrm{~min}$ of administration. Corresponding alterations to indices of mitochondrial, cytosolic and whole-cell redox potential were observed. Pyruvate oxidation was maintained in the diabetic rats,
\end{abstract}

Electronic supplementary material The online version of this article (https://doi.org/10.1007/s00125-017-4445-6) contains peer-reviewed but unedited supplementary material, which is available to authorised users.

Christoffer Laustsen

cl@ clin.au.dk

1 MR Research Centre, Department of Clinical Medicine, Aarhus University, Palle Juul-Jensens Boulevard 99, 8200 Aarhus N, Denmark

2 Department of Medical Cell Biology, Uppsala University, Uppsala, Sweden suggesting that the diabetic state abrogates metabolic reprogramming caused by metformin.

Conclusions/interpretation This study demonstrates that metformin-induced acute metabolic alterations in healthy kidneys favoured anaerobic metabolism at the expense of aerobic metabolism. The results suggest that metformin directly alters the renal redox state, with elevated renal cytosolic redox states as well as decreased mitochondrial redox state. These findings suggest redox biology as a novel target to eliminate the renal complications associated with metformin treatment in individuals with impaired renal function.

Keywords Diabetes · Hyperpolarised MRI · Metformin · Renal function $\cdot$ Renal metabolism $\cdot$ Renal redox

$\begin{array}{ll}\text { Abbreviations } \\ \text { AMPK } & \text { AMP-activated protein kinase } \\ \text { LDH } & \text { Lactate dehydrogenase } \\ \text { MAP } & \text { Mean arterial pressure } \\ \text { mGPD } & \text { Mitochondrial glycerophosphate dehydrogenase } \\ \mathrm{PDH} & \text { Pyruvate dehydrogenase } \\ \mathrm{pO}_{2} & \text { Oxygen tension } \\ \mathrm{Q}_{\mathrm{O} 2} & \text { Oxygen consumption } \\ \mathrm{RBF} & \text { Renal blood flow } \\ \mathrm{RVR} & \text { Renal vascular resistance } \\ \mathrm{STZ} & \text { Streptozotocin } \\ \mathrm{T}_{\mathrm{Na}} & \text { Tubular Na }{ }^{+} \text {reabsorption }\end{array}$

\section{Introduction}

Metformin remains the primary candidate to promote glycaemic control in individuals with type 2 diabetes [1], yet its mechanism of action is still not understood. Metformin 
belongs to the biguanide class of molecules, oral glucoselowering drugs that increase insulin sensitivity rather than invoke altered insulin production. The increased insulin sensitivity reduces plasma glucose levels due to increased glucose uptake, glucose disposal and decreased gluconeogenesis [2-4]. Moreover, it has been proposed that a major effect of metformin is inhibition of gluconeogenesis in individuals with type 2 diabetes [5]. Metformin was recently reported to inhibit the mitochondrial form of glycerophosphate dehydrogenase (mGPD) in the liver, thus decreasing hepatic gluconeogenesis and altering the cytosolic and mitochondrial redox state [2].

Although metformin is the preferred drug in individuals with type 2 diabetes, its use in type 1 diabetes has been limited. Preliminary evidence has suggested that metformin also reduces the need for insulin therapy in individuals with insulinopenic diabetes [3,6], and recent and ongoing clinical trials have investigated combined therapy with metformin and insulin in type 1 diabetic participants [7, 8]. Furthermore, in recent years, the potential anticancer action of metformin has also attracted increasing interest [9-14]; it has been postulated that this action is mediated by activation of the AMP-activated protein kinase (AMPK) pathway and concomitant suppression of cyclic AMP formation.

A potentially serious side effect of metformin treatment is lactic acidosis, which has limited its application for the treatment of diabetes in individuals who also have kidney disease [15]. Lactic acidosis is characterised by elevated plasma lactate concentrations of more than $5 \mathrm{mmol} / \mathrm{l}$, decreased blood $\mathrm{pH}$ to less than 7.35 and an increased anion gap [16]. A plausible mechanism for the induction of lactic acidosis by metformin involves inhibition of mitochondrial glycerol-3phosphate dehydrogenase, leading to an increase in the cytosolic redox state; this results in reduced conversion of lactate by the liver to pyruvate, and increased systemic lactate concentrations [2]. This mechanism of an altered redox state and increased systemic lactate has been verified in the liver and heart $[2,17]$.

In the kidney, the link between redox state, systemic lactate and metabolic acidosis in the presence of metformin has not yet been explored in detail. This is of particular interest in relation to the kidneys in diabetes, where an already altered redox state (decreased $\mathrm{NAD}^{+} / \mathrm{NADH}$ ratio) is already apparent, along with increased gluconeogenesis [18-20]. Experimental research and studies of participants with diabetic nephropathy have identified a correlation between metabolic acidosis, renal insufficiency and altered renal oxygen metabolism [21-23].

In this study, we investigated the acute metabolic response of healthy and insulinopenic rats, used as a model of diabetes, to metformin, using a combination of invasive techniques and non-invasive hyperpolarised magnetic resonance metabolic imaging $[18,24,25]$. We used a sensitive non-invasive metabolic imaging method that relies on the ability to trace the metabolic conversion of intravenously injected nonradioactive metabolic biomarkers, such as $\left[1-{ }^{13} \mathrm{C}\right]$ pyruvate, which undergo metabolic conversion into metabolic derivatives such $\left[1-{ }^{13} \mathrm{C}\right]$ lactate, $\left[1-{ }^{13} \mathrm{C}\right]$ alanine and ${ }^{13} \mathrm{CO}_{2} /{ }^{13} \mathrm{C}$-bicarbonate [26-28]. Our aim was to investigate the effect of metformin treatment on redox balance and interactions with anaerobic and aerobic metabolic pathways in in vivo metabolism in kidneys from healthy and diabetic animals.

\section{Methods}

Animal model and acute treatment The study complied with the guidelines for the use and care of laboratory animals and was approved by the Danish Inspectorate of Animal Experiments. All rats included in this study were 6-7-week old Wistar rats (HanTac:WH; Taconic, Ry, Denmark) with a weight of 200 (range 180-230) g. All animals had free access to water and standard chow throughout the study. The rats were kept in cages with a $12 \mathrm{~h} / 12 \mathrm{~h}$ light/dark cycle, a temperature of $21 \pm 2{ }^{\circ} \mathrm{C}$ and humidity of $5 \pm 5 \%$. They were randomly grouped into a diabetic group, which received streptozotocin (STZ) injection, and a healthy control group, which only received buffer. Anaesthesia in the animals was induced (7\% sevoflurane in $21 / \mathrm{min}$ air [vol./vol.]) and maintained (3\% sevoflurane in $2 \mathrm{l} / \mathrm{min}$ air) by sevoflurane via spontaneously respiration, and adequate depth of anaesthesia was determined by the pedal reflex.

Insulinopenic diabetes was induced as previously described $[29,30]$. In brief, rats received freshly prepared STZ ( $55 \mathrm{mg} / \mathrm{kg}$ body weight; Sigma-Aldrich, Brøndby, Denmark) in sodium citrate buffer. Blood glucose was measured in tail capillary blood using a Contour blood glucose meter (Bayer Diabetic Care, Copenhagen, Denmark). Rats were considered diabetic when blood glucose levels exceeded $15 \mathrm{mmol} / 148 \mathrm{~h}$ after injection of STZ. The diabetic rats did not receive insulin or any glucose-lowering medicine apart from metformin. Metformin (total volume $0.2-0.3 \mathrm{ml}, 50 \mathrm{mg} / \mathrm{kg}$ body weight in $0.9 \%$ saline $[154 \mathrm{mmol} / 1 \mathrm{NaCl}]$ ), or volume-matched vehicle, was administered intravenously as a bolus via a tail vein catheter.

Invasive measurements of kidney function Invasive measurements of kidney oxygen homeostasis were performed using Clark-type electrodes in 11 healthy rats before and 30 min after metformin treatment. GFR, renal blood flow (RBF), mean arterial pressure (MAP), renal vascular resistance (RVR), tubular $\mathrm{Na}^{+}$reabsorption $\left(\mathrm{T}_{\mathrm{Na}}\right)$, tubular oxygen consumption $\left(\mathrm{Q}_{\mathrm{O} 2}\right)$ and cortical and medullary oxygen tension $\left(p \mathrm{O}_{2}\right)$ were measured as previously described [24, 25].

In brief, animals were anaesthetised with Inactin (sodium thiobutabarbital i.p., $120 \mathrm{mg} / \mathrm{kg}$ body weight; Sigma-Aldrich, St. Louis, MO, USA) and placed on a servo-rectally controlled 
heating pad to maintain body temperature at $37^{\circ} \mathrm{C}$. Animals were tracheotomised, and a catheter was placed in the urinary bladder to allow drainage. Catheters were inserted into the left femoral vein for infusion of saline $\left(5 \mathrm{ml} \mathrm{kg}\right.$ body weight ${ }^{-1} \mathrm{~h}^{-1}$ ) and left femoral artery for measurement of blood pressure and blood sampling. The left kidney was exposed by a flank incision and immobilised in a plastic cup. Urine was collected from a catheter placed in the left ureter, and a venous blood sample was taken from the left renal vein at the end of the experiment to determine arteriovenous extraction of oxygen.

Surgery was followed by a 40 min recovery period and a 60 min experimental period during which GFR was measured by ${ }^{3} \mathrm{H}$-inulin clearance and RBF by a flow probe place around the left renal artery. The activity of ${ }^{3} \mathrm{H}$ in plasma and urine was measured using standard liquid scintillation technique. Blood gas variables in samples from the left renal vein and femoral artery were analysed (i-STAT; Abbott Point of Care, Princeton, NJ, USA). Kidney cortex and medullary $p \mathrm{O}_{2}$ were measured, at $1 \mathrm{~mm}$ and $3 \mathrm{~mm}$ depth from the kidney surface, respectively, using a Clark-type microelectrode (OXY-10; Unisense A/S, Aarhus, Denmark). $p \mathrm{O}_{2}$ was measured over $30-60 \mathrm{~s}$ in five different locations for cortex and medulla, respectively, and averaged into one mean $(n=1$ per animal and time).

Hyperpolarised MRI Twenty-one 6-7-week old Wistar rats (Taconic) with a weight of 200 (range 180-230) g were included in this study. The hyperpolarised MRI examinations were performed as previously described $[31,32]$ on a $3 \mathrm{~T}$ clinical MR system (GE Healthcare, Waukesha, WI, USA) equipped with a dual-tuned ${ }^{13} \mathrm{C} /{ }^{1} \mathrm{H}$ volume rat coil (GE Healthcare, Brøndby, Denmark) using a slice-selective ${ }^{13} \mathrm{C}$ iterative decomposition of water and fat with echo asymmetry and last-squares estimation (IDEAL) spiral sequence. The flip angle was $10^{\circ}$, there were 11 IDEAL echoes and one initial spectrum per IDEAL encoding, repetition time (TR)/echo time (TE) $/ \Delta \mathrm{TE}$ values were $100 \mathrm{~ms} / 0.9 \mathrm{~ms} / 0.9 \mathrm{~ms}$, and field of view (FOV) was $80 \mathrm{~mm} \times 80 \mathrm{~mm}$, with $5 \mathrm{~mm} \times 5 \mathrm{~mm}$ real resolution and an axial oblique slice thickness of $15 \mathrm{~mm}$ covering both kidneys. Three rats did not develop sufficient diabetes (blood glucose $<15 \mathrm{mmol} / \mathrm{l}$ continuously $48 \mathrm{~h}$ after STZ injection), two diabetic rats were excluded owing to complications of diabetes, and two control rats were excluded because of technical problems with the scanner and hyperpolariser. Thus, nine diabetic rats (three female and six male rats) and six female control rats received metformin treatment, and six male control rats received saline and successfully completed the MRI examination.

In brief, 12 healthy and nine diabetic rats ( 4 weeks after STZ induction) were anaesthetised (3\% sevoflurane in $21 /$ min air) and a tail vein catheter $(0.4 \mathrm{~mm})$ was inserted for injection of metformin or saline and administration of hyperpolarised $\left[1-{ }^{13} \mathrm{C}\right]$ pyruvate polarised in a SpinLab
(GE Healthcare, Brøndby, Denmark). Each animal was injected twice with $1.5 \mathrm{ml}(125 \mathrm{mmol} / \mathrm{l})$ hyperpolarised $\left[1-{ }^{13} \mathrm{C}\right]$ pyruvate, before and $30 \mathrm{~min}$ after administration of metformin or saline (Fig. 1 shows study design). All hyperpolarised $\left[1-{ }^{13} \mathrm{C}\right]$ pyruvate samples were balanced to a $\mathrm{pH}$ value of around 7.4 by the addition of neutralisation buffer. After the MRI hyperpolarisation scans, arterial blood electrolytes values for each rat were obtained using an i-STAT blood analyser immediately after blood sampling from the aortic bifurcation.

Redox assays An additional 16 healthy rats were randomly divided into a control group (eight rats) that received saline, and a metformin group (eight rats) that received metformin for $30 \mathrm{~min}$. Blood, urine and freeze-clamped kidney tissue were harvested from these rats for subsequent systolic redox assays and AMPK analyses. In brief, 5-7 ml arterial blood samples were collected from the aortic bifurcation, and kidney cortical tissue was freeze-clamped and harvested. Lactate/pyruvate and $\mathrm{NAD}^{+} / \mathrm{NADH}$ were measured as indices of cytosolic and whole-cell redox potential, in addition to the mitochondrial redox assay $\beta$-hydroxybutyrate/acetoacetate, as previously described [31, 32].

\section{Protein isolation and semi-quantitative immunoblotting}

Renal cortex tissue was freeze-clamped and homogenised in dissecting buffer $(0.3 \mathrm{~mol} / \mathrm{l}$ sucrose, $25 \mathrm{mmol} / \mathrm{l}$ imidazole, $1 \mathrm{mmol} / \mathrm{l}$ EDTA, $\mathrm{pH}$ 7.2) containing the following protease inhibitors: Phosphatase Inhibitor Cocktails 2 and 3 (Sigma-Aldrich, St Louis, MO, USA) and Complete Mini Protease Inhibitor Cocktail Tablets (serine, cysteine and metalloprotease inhibitor; Thermo Scientific, Hvidovre, Denmark). The tissue was homogenised for $240 \mathrm{~s}$ at $50 \mathrm{~Hz}$ by a TissueLyser LT (Qiagen, Hilden, Germany) and then centrifuged at $1000 \mathrm{~g}$ at $4^{\circ} \mathrm{C}$ for $10 \mathrm{~min}$. Gel samples were prepared from the supernatant fraction in Laemmli sample buffer containing $2 \% \mathrm{SDS}$ (wt/vol.). The total protein concentration of the homogenate was measured using a Pierce BCA protein assay kit (Thermo Scientific).

Protein samples were run on $12 \%$ Criterion TGX Precast Gel (Bio-Rad Laboratories, Copenhagen, Denmark). Proteins were transferred to a nitrocellulose membrane (Hybond ECL; GE Healthcare, Hatfield, UK). The blots were then blocked with 5\% non-fat dry milk (wt/ vol.) in TBS-T $(20 \mathrm{mmol} / \mathrm{l}$ Tris-Base, $137 \mathrm{mmol} / \mathrm{l} \mathrm{NaCl}$, $0.1 \%$ Tween-20 [vol./vol.], adjusted to $\mathrm{pH}$ 7.4). After washing with TBS-T, the blots were incubated with primary antibodies (AMPK and Thr172-phospho-AMPK; Cell Signaling, Leiden, the Netherlands) overnight at $4^{\circ} \mathrm{C}$. The antigen-antibody complex was visualised with horseradish peroxidase-conjugated secondary antibodies (goat antirabbit P448, diluted 1:4000; Dako; Glostrup, Denmark) for $1 \mathrm{~h}$ at room temperature using enhanced 


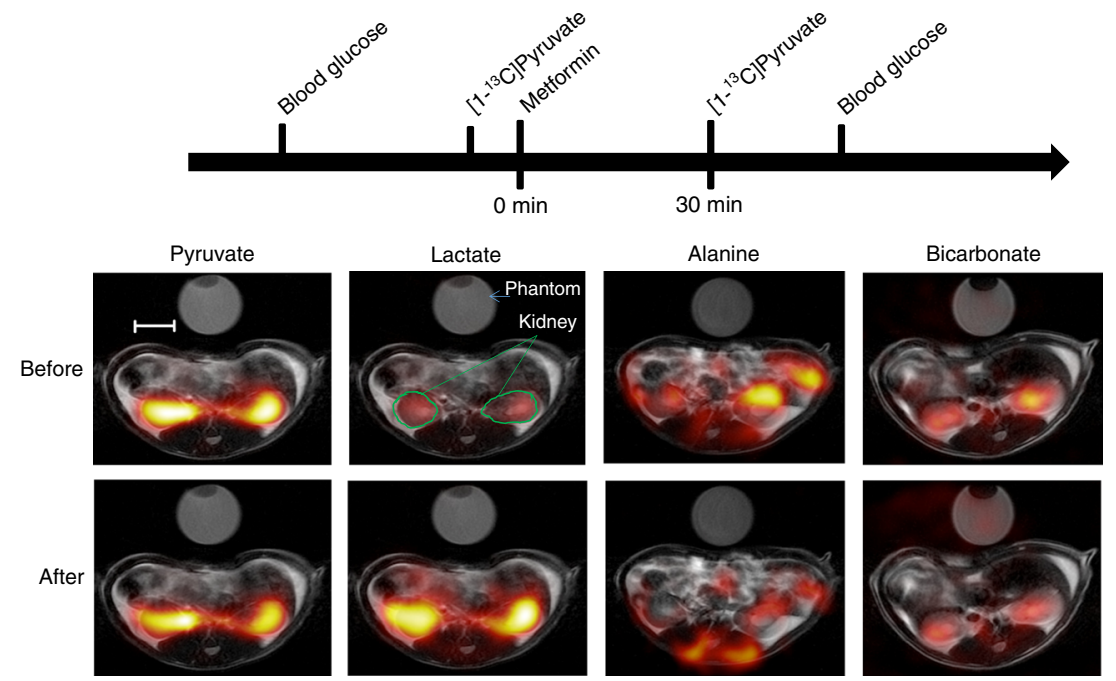

Fig. 1 Study design. Rats in a healthy control group and a diabetic group underwent two consecutive hyperpolarised $\left[1-{ }^{13} \mathrm{C}\right]$ pyruvate examinations before and 30 min after metformin treatment. Representation of anatomical renal ${ }^{1} \mathrm{H}$-MRI images overlaid with ${ }^{13} \mathrm{C}$ images before and after

chemiluminescence system (ECL, Amersham ECL Plus; GE Healthcare, Brøndby, Denmark). All western blots were normalised to total protein, as measured using Stain-Free technology.

Statistics All data are presented as means \pm SEM. All statistical analysis was performed using GraphPad Prism 7 (Graphpad Software; La Jolla, CA, USA). Data were analysed by a two-tailed Student's $t$ test with normality (Shapiro-Wilk normality test, $p>0.1$ ) for comparison of animal and kidney weight, blood glucose and plasma data, as well as redox data and AMPK activation data between two different groups. A paired $t$ test was used to compare the effect of metformin treatment in the same group for hyperpolarised ${ }^{13} \mathrm{C}$ data and renal physiological effect. Repeated measures two-way ANOVA was used to compare the effect of metformin treatment between the healthy and diabetic groups, with Sidak's multiple comparisons test testing the effect before and after metformin treatment in the same group. A value of $p<0.05$ was considered statistically significant.

\section{Results}

Physiological effects of metformin on the healthy kidney Experiments performed with invasive Clark-electrodes measured renal physiological variables before and after metformin treatment. The GFR did not change ( $p=0.79$; Fig. 2a). However, RBF increased 1.2-fold after metformin treatment (Fig. 2b), meaning that $\mathrm{O}_{2}$ delivery was increased. This was reflected by a significant increase in cortex tissue $\mathrm{pO}_{2}$ $\left(p<0.001\right.$; Fig. 2h) and medullary $\mathrm{OO}_{2}(p=0.03$; Fig. $2 \mathrm{i})$. metformin treatment in healthy control rats, showing an axial slab of $\left[{ }^{13} \mathrm{C}\right]$ pyruvate, $\left[{ }^{13} \mathrm{C}\right]$ lactate, $\left[{ }^{13} \mathrm{C}\right]$ alanine and ${ }^{13} \mathrm{C}$-bicarbonate. Strong $\left[{ }^{13} \mathrm{C}\right]$ lactate and decreased ${ }^{13} \mathrm{C}$-bicarbonate signals were seen in the post-treatment kidney. Scale bar, $10 \mathrm{~mm}$

Metformin administration also decreased RVR, by $16 \%$ (Fig. $2 \mathrm{~d}$ ). No changes were observed in MAP ( $p=0.19$; Fig. 2c), $\mathrm{T}_{\mathrm{Na}}(p=0.71$; Fig. $2 \mathrm{e}), \mathrm{Q}_{\mathrm{O} 2}(p=0.85$; Fig. $2 \mathrm{~g})$ or $\mathrm{T}_{\mathrm{Na}} / \mathrm{Q}_{\mathrm{O} 2}$ $(p=0.43$; Fig. 2f) with metformin treatment.

Metabolic effects of metformin on the healthy kidney Metformin treatment changed both anaerobic metabolism $\left(\left[1-{ }^{13} \mathrm{C}\right]\right.$ lactate/[1- $\left.{ }^{13} \mathrm{C}\right]$ pyruvate ratio increased 2.0-fold; Fig. $3 \mathrm{a})$ and aerobic metabolism $\left({ }^{13} \mathrm{C}\right.$-bicarbonate/ $\left[1-{ }^{13} \mathrm{C}\right]$ pyruvate ratio decreased by $16 \%$; Fig. $3 \mathrm{c}$ ) in kidneys from healthy animals. A dramatic decrease in intracellular metabolite distribution was seen ([1- $\left.{ }^{13} \mathrm{C}\right]$ alanine/ $\left[1-{ }^{13} \mathrm{C}\right]$ pyruvate ratio was decreased by $47 \%$; Fig. $\left.3 \mathrm{~b}\right)$. Furthermore, acute metformin treatment produced a 2.3-fold increase in the balance between anaerobic and aerobic metabolism ([1- $\left.{ }^{13} \mathrm{C}\right]$ lactate $/{ }^{13} \mathrm{C}$-bicarbonate ratio; Fig. $\left.3 \mathrm{~d}\right)$. Moreover, to verify that the renal metabolic changes resulted from metformin treatment, and were not an artefact caused by the large pyruvate injection, six rats were given saline infusion as a placebo treatment. No metabolic effects could be detected in any of the renal metabolic pathways examined in the six rats that received placebo (see electronic supplementary material [ESM] Fig. 1).

Redox effects of metformin on the healthy kidney Neither plasma lactate concentration (control group $4.0 \pm 1.9 \mathrm{mmol} /$ 1 , metformin group $5.3 \pm 1.3 \mathrm{mmol} / \mathrm{l} ; p=0.59$ ) nor plasma pyruvate concentration (control group $0.90 \pm 0.23 \mathrm{mmol} / \mathrm{l}$, metformin group $1.35 \pm 0.30 \mathrm{mmol} / \mathrm{l} ; p=0.24$ ) showed a significant difference between metformin-treated and control rats. However, within the kidney cortex, lactate concentration increased 5.2-fold in metformin-treated rats 
a

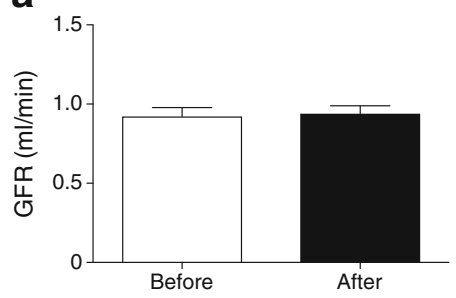

d

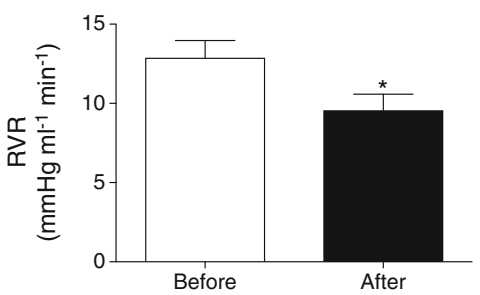

g

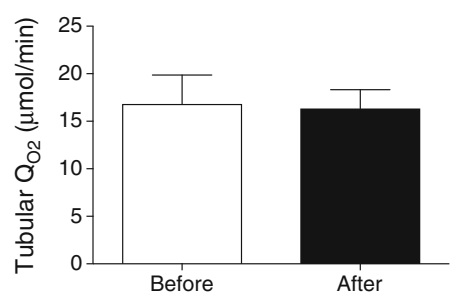

b

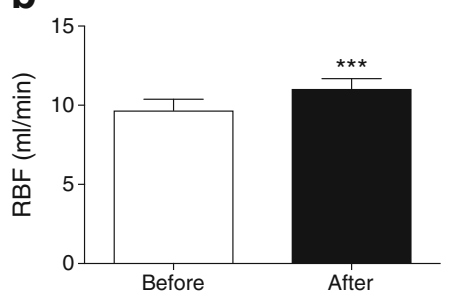

e

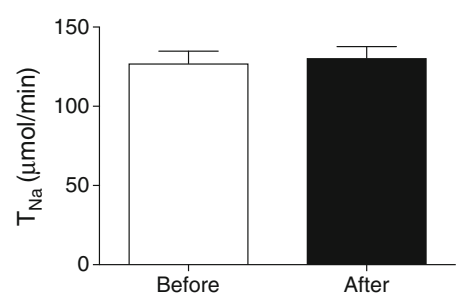

h

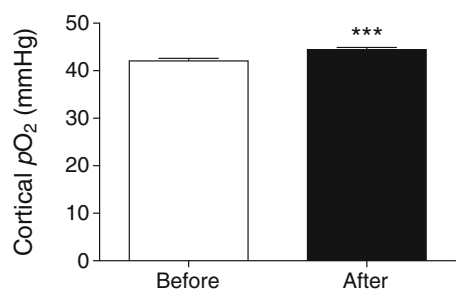

C

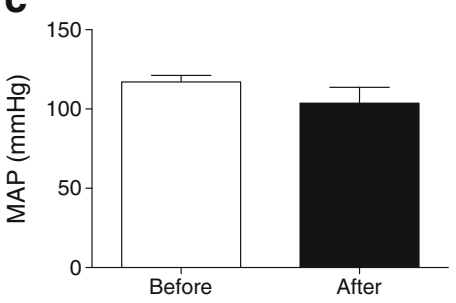

f

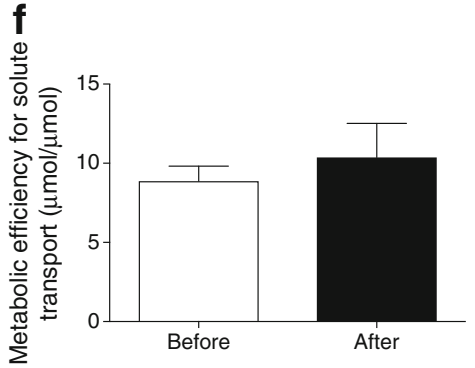

i

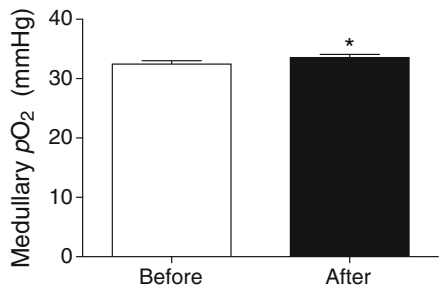

Fig. 2 Renal physiological variables were measured invasively before and after metformin treatment in healthy rats. (a) GFR. (b) RBF. (c) MAP. (d) RVR. (e) $\mathrm{T}_{\mathrm{Na}}$. (f) Metabolic efficiency for solute transport. (g) Tubular

compared with saline-treated control rats (Fig. 4a). Cortical pyruvate concentration showed no difference between the treated and untreated groups (Fig. 4b). Thus, the lactate/ pyruvate ratio increased 5.4-fold in metformin-treated compared with control rats (Fig. 4c). Moreover, metformin
Qo2. (h) Cortical $p \mathrm{O}_{2}$. (i) Medullary $p \mathrm{O}_{2} . n=11$ per group; ${ }^{*} p<0.05$, *** $p<0.001$ for measurement after vs before metformin treatment

treatment decreased the concentration of $\beta$ hydroxybutyrate in the kidney cortex by $44 \%$ (Fig. 4d), while the concentration of acetoacetate was maintained (Fig. 4e). Thus, metformin treatment reduced the $\beta$ hydroxybutyrate/acetoacetate ratio by $37 \%$ (Fig. 4 f). The
Fig. 3 Alterations in metabolic pathways in the kidneys of healthy rats before and $30 \mathrm{~min}$ after metformin treatment. (a) Lactate/pyruvate ratio. (b) Alanine/pyruvate ratio. (c) Bicarbonate/pyruvate ratio. (d) Lactate/bicarbonate ratio. $n=6$ per group; $* p<0.05$,

$* * * p<0.001$ for measurement after vs before metformin treatment a
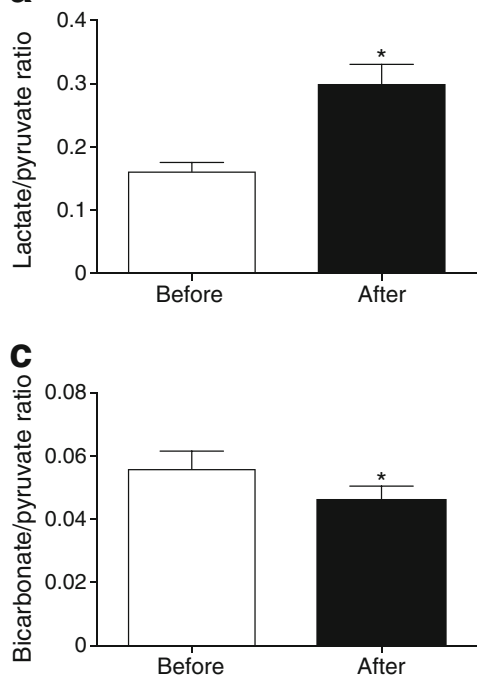

b

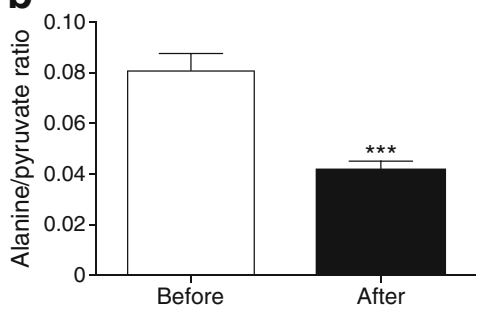

d

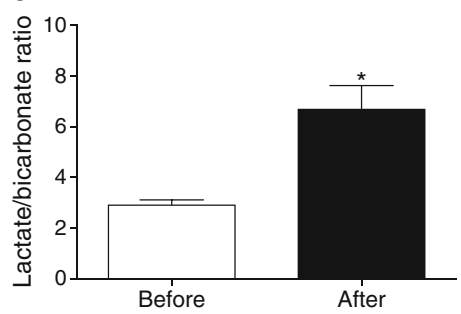




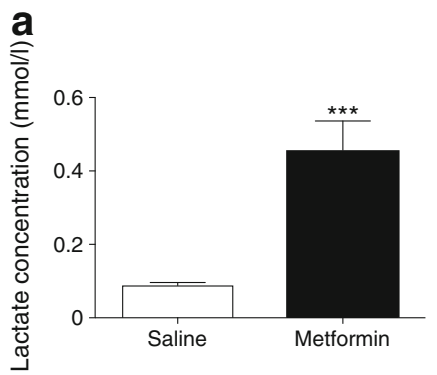

b

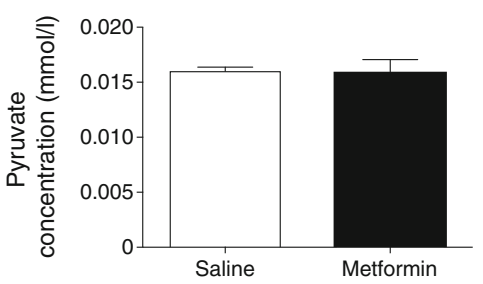

e
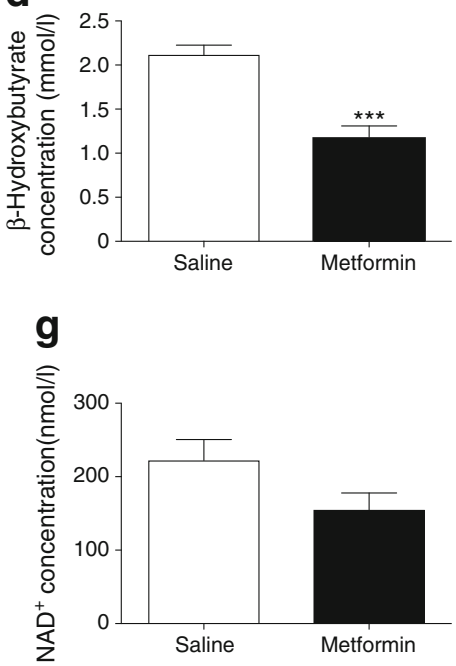

C

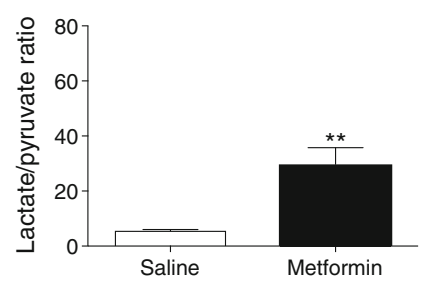

f

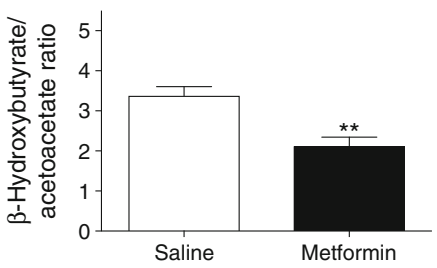

i

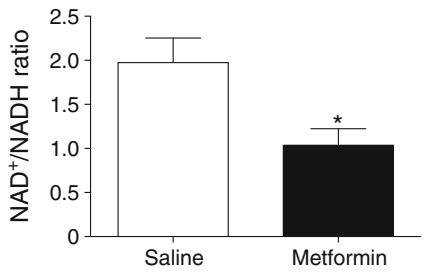

Fig. 4 Changes in renal cytosolic redox states and renal mitochondrial redox states in healthy kidney cortex in the metformin treatment group compared with the saline control group. (a) Lactate concentration. (b) Pyruvate concentration and (c) lactate/pyruvate concentration ratio. (d) $\beta$-Hydroxybutyrate concentration, (e) acetoacetate concentration and (f) $\beta$-hydroxybutyrate/acetoacetate concentration ratio. (g) $\mathrm{NAD}^{+}$concentration, (h) $\mathrm{NADH}$ concentration and (i) $\mathrm{NAD}^{+} / \mathrm{NADH}$ concentration ratio. $n=8$ per group; ${ }^{*} p<0.05,{ }^{*} p<0.01$, *** $p<0.001$ for metformin vs saline total NAD concentration in the renal cortex did not differ between metformin-treated rats $(310 \pm 27 \mathrm{nmol} / \mathrm{l})$ and control group rats $(338 \pm 31 \mathrm{nmol} / 1 ; p=0.52)$. The $\mathrm{NAD}^{+}$ concentration was not different between the groups ( $p=0.09$; Fig. $4 \mathrm{~g}$ ); however, the NADH concentration was significantly increased (1.3-fold) in the metformintreated compared with control rats (Fig. 4h). This resulted in a decrease in the $\mathrm{NAD}^{+} / \mathrm{NADH}$ concentration ratio of $47 \%$ following acute metformin treatment (Fig. 4i).

Metformin increased AMPK activation in the healthy kidney Treatment with metformin for $30 \mathrm{~min}$ did not change the total protein level of AMPK ( $p=0.63$; Fig. $5 b)$, but increased the p-AMPK protein level 2.4-fold (Fig. 5a).

Metabolic reprogramming with metformin in healthy vs type 1 diabetic rats All the diabetic rats included developed sustained hyperglycaemia, averaging $31.4 \pm 1.4 \mathrm{mmol} / \mathrm{l} \mathrm{com}-$ pared with $6.9 \pm 0.1 \mathrm{mmol} / \mathrm{l}$ in control rats. Diabetic rats had a lower body weight and higher kidney weight than control rats $(p<0.05$; Table 1).
The arterial blood analysis using the i-STAT showed that blood sodium concentrations did not differ between the diabetic group and the control group $(p=0.16)$. Potassium and total carbon dioxide were elevated in the diabetic group, while chloride and free calcium levels were decreased in the diabetic group ( $p<0.05$ for all comparisons; Table 2). Blood urea nitrogen was significantly increased in diabetic rats $(p<0.05)$, while blood creatinine was decreased $(p<0.05)$ in the diabetic group compared with the control rats. No difference was observed between controls and diabetic rats in the state of anion gap metabolic acidosis (Table 2).

In all pathways investigated using hyperpolarised examinations, acute metformin treatment elicited different responses in control rats compared with diabetic rats receiving metformin treatment (Fig. 6). In healthy control rats, metformin treatment led to an increase in the $\left[1-{ }^{13} \mathrm{C}\right]$ lactate/ $\left[1-{ }^{13} \mathrm{C}\right]$ pyruvate ratio $(p=0.003)$, accompanied by reductions in the $\left[1-{ }^{13} \mathrm{C}\right]$ alanine/ $\left[1-{ }^{13} \mathrm{C}\right]$ pyruvate ratio $(p<0.0001)$ (Fig. $6 \mathrm{a}, \mathrm{b})$. The ${ }^{13} \mathrm{C}$-bicarbonate/ $\left[1-{ }^{13} \mathrm{C}\right]$ pyruvate ratio did not show a significant difference between healthy rats and diabetic rats (Fig. 6c). Moreover, 
Fig. 5 Effects of metformin treatment on AMPK phosphorylation. (a) p-AMPK/ total protein. (b) AMPK/total protein. $n=8$ per group $* p<0.05$ for metformin vs saline a

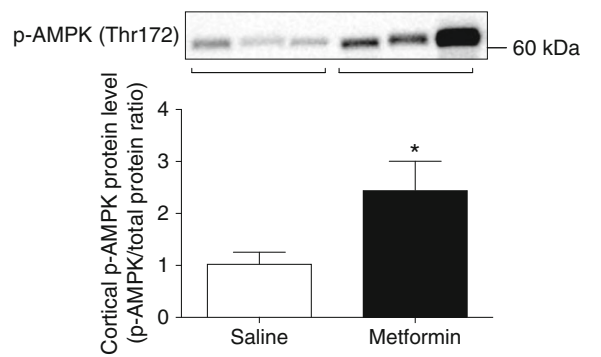

b

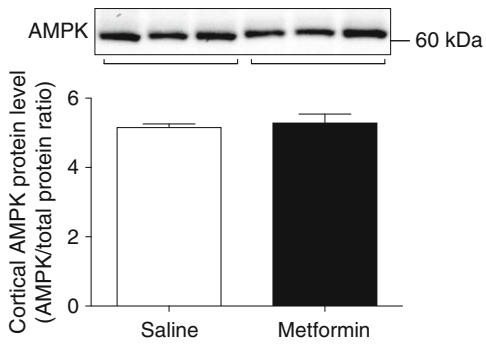

acute metformin treatment in healthy control rats compared with diabetic rats increased the balance between anaerobic and aerobic metabolism $\left(\left[1-{ }^{13} \mathrm{C}\right]\right.$ lactate $/{ }^{13} \mathrm{C}$-bicarbonate ratio) (Fig. 6d).

\section{Discussion}

Our findings further support the proposed mechanisms of metformin's action in lowering blood glucose described by Madiraju et al [2], in which metformin inhibits $\mathrm{MGPD}$ in liver, thus increasing the cytosolic redox state, decreasing the mitochondrial redox state and hepatic gluconeogenesis, and increasing AMPK activation.

The main finding in this study is that, in the healthy kidney, metformin causes an acute energetic shift away from aerobic metabolism to anaerobic metabolism (i.e. the Pasteur effect). Acute metformin treatment increased lactate dehydrogenase (LDH) flux and inhibited pyruvate dehydrogenase (PDH) flux via an altered cytosolic and mitochondrial redox state, directly limiting the substrates available to drive gluconeogenesis. This change is largely blunted in the kidney tissue in diabetes, where a basal increased lactate concentration is seen. Interestingly, the effects were observed in the presence of increased RBF, which supplied more oxygen to the tissue and increased cortical $p \mathrm{O}_{2}$. Therefore the observed shift in substrate metabolism was independent of oxygen.

Table 1 Body weight, kidney weight, blood glucose level and kidney weight per $\mathrm{kg}$ of rat weight on the day of the scan in control and diabetic rats

\begin{tabular}{lll}
\hline Variable & Control & Diabetes \\
\hline Body weight $(\mathrm{g})$ & $289 \pm 6$ & $220 \pm 8^{*}$ \\
Kidney weight $(\mathrm{g})$ & $1.03 \pm 0.06$ & $1.23 \pm 0.03^{*}$ \\
Blood glucose level (mmol/l) & $6.9 \pm 0.1$ & $31.4 \pm 1.4^{*}$ \\
Kidney weight/body weight $(\mathrm{g} / \mathrm{kg})$ & $3.6 \pm 0.2$ & $5.6 \pm 0.3^{*}$ \\
\hline
\end{tabular}

Data are mean \pm SEM of $n=4-7$ per group

Kidney weight was the mean of both kidneys

Blood glucose was measured in the fed state

$* p<0.05$ vs control group
In addition renal GFR, $\mathrm{Q}_{\mathrm{O} 2}, \mathrm{~T}_{\mathrm{Na}}$ and $\mathrm{T}_{\mathrm{Na}} / \mathrm{Q}_{\mathrm{O} 2}$ were largely unaltered, indicative of maintained renal function and oxygen demand. This finding suggests that the reduction in PDH flux we observed upon metformin treatment did not reflect a localised change in renal energy metabolism per se. Rather, it is likely that metformin reduced renal PDH flux owing to its systemic redox effects, the accumulation of NADH, which is unfavourable for the conversion of lactate to pyruvate, directly inhibiting pyruvate oxidation (limiting availability of $\mathrm{NAD}^{+}$). This is supported by metformin treatment leading to a reduction in alanine signal, a pool size that represents the intracellular pyruvate pool and thus indicates a simultaneous shift in the pyruvate to lactate pool size, as seen in the biochemical analysis.

The increased anaerobic metabolic pathway (indicated by $\left[1-{ }^{13} \mathrm{C}\right]$ lactate production) was similarly increased in previous reports on the liver and heart $[2,17]$. Analyses of freezeclamped kidney tissue showed that metformin increased lactate concentration and lactate/pyruvate ratio, which corresponded with the results of the hyperpolarised MRI. Moreover, we demonstrated that acute metformin treatment changed the renal redox state, elevating cytosolic redox potential and decreasing mitochondrial redox potential. A similar effect has previously been demonstrated in liver and heart, although this, contrary to

Table 2 Arterial blood electrolytes and characteristics in control and diabetic rats

\begin{tabular}{lll}
\hline Variable & Control & Diabetic \\
\hline $\mathrm{Na}^{+}(\mathrm{mmol} / \mathrm{l})$ & $137 \pm 0$ & $137 \pm 0$ \\
$\mathrm{~K}^{+}(\mathrm{mmol} / \mathrm{l})$ & $3.4 \pm 0.1$ & $3.9 \pm 0.1^{*}$ \\
$\mathrm{Cl}^{-}(\mathrm{mmol} / \mathrm{l})$ & $100 \pm 0$ & $93 \pm 2^{*}$ \\
$\mathrm{Ca}^{2+}(\mathrm{mmol} / \mathrm{l})$ & $1.40 \pm 0.02$ & $1.24 \pm 0.04^{*}$ \\
$\mathrm{Total} \mathrm{CO}_{2}(\mathrm{mmol} / \mathrm{l})$ & $23.0 \pm 0.7$ & $28.5 \pm 0.3^{*}$ \\
Blood urea nitrogen $(\mathrm{mmol} / \mathrm{l})$ & $5.46 \pm 0.1$ & $9.64 \pm 0.8^{*}$ \\
Creatinine (mmol/l) & $32.84 \pm 1.6$ & $17.46 \pm 0.2^{*}$ \\
Haematocrit $(\%)$ & $34.9 \pm 0.9$ & $35.8 \pm 1.1$ \\
Haemoglobin $(\mathrm{g} / \mathrm{l})$ & $118.4 \pm 3.1$ & $121.5 \pm 3.8$ \\
Anion gap (mmol/l) & $18.3 \pm 0.5$ & $20.5 \pm 1.3$ \\
\hline
\end{tabular}

Data are mean \pm SEM of $n=4-7$ per group

$* p<0.05$ vs control group 

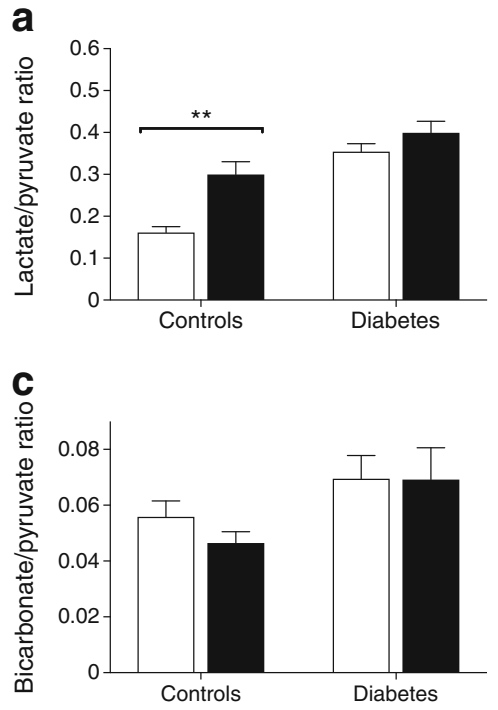

Fig. 6 Comparison of metabolic pathway alterations between kidney tissue from healthy control and diabetic rats at baseline (white bars) and after acute bolus administration of metformin (black bars). (a) Lactate/ pyruvate ratio ( $p=0.0001$ for group, $p=0.0012$ for treatment and $p=0.0562$ for interaction). (b) Alanine/pyruvate ratio $(p=0.134$ for

our results, found a maintained total cellular $\mathrm{NAD}^{+} / \mathrm{NADH}$ redox state, balanced by the subcellular redox changes $[2,17]$. It is important to note that our reported changes are acute, and prior reports have emphasised that chronic metformin treatment inhibits complex 1 mitochondrial respiration [33, 34]. However inhibition of complex 1 is generally seen at high concentrations above $1 \mathrm{mmol} / \mathrm{l}$, which is $10-20$ times the clinical dose that was used in this study $[2,33,34]$.

Our study showed a decrease in the $\mathrm{NAD}^{+} / \mathrm{NADH}$ ratio, which means that the total cellular redox state and energy charge in the kidney had a different response to metformin than was seen with the liver and heart. This could be explained by the use of intravenous administration for the metformin, thereby increasing the local accumulation of metformin in the kidneys and driving the concentration-dependent mitochondrial respiratory inhibition [2, 34, 35]. This is, however, unlikely as the systemic pharmacokinetic responses to acute $50 \mathrm{mg} / \mathrm{kg}$ intravenous metformin are consistent with those of oral treatment in individuals with diabetes, and with previous reports of the effects of acute and chronic treatment on the liver and heart $[2,17]$.

Good glycaemic control of diabetes and early effective treatment of diabetes-associated complications are important in preventing or delaying nephropathy [21]. The role of metformin in maintaining glycaemic control in individuals with renal impairment has, however, been questioned, owing to a purported association between metformin treatment and lactate acidosis. This has been proposed to originate from decreased gluconeogenesis leading to increased anaerobic metabolism and an altered redox state, and subsequent inhibition of mitochondrial respiration $[21,22,36]$. b
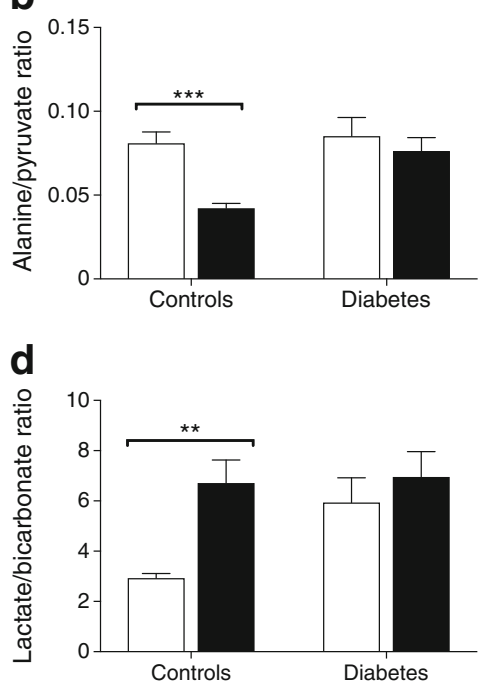

group, $p=0.0001$ for treatment and $p=0.0044$ for interaction). (c) Bicarbonate/pyruvate ratio ( $p=0.13$ for group, $p=0.46$ for treatment and $p=0.49$ for interaction). (d) Lactate/bicarbonate ratio ( $p=0.21$ for group, $p=0.0007$ for treatment and $p=0.0246$ for interaction). $n=6$ for control rats; $n=9$ for diabetic rats; ** $p<0.01$, *** $p<0.001$ as shown

Metabolic acidosis is recognised as a risk factor in those with chronic kidney disease, with low bicarbonate concentration being associated with worsening effects on the progression of chronic kidney disease [37]. Interestingly, a metformin overdose can largely decrease global $\mathrm{Q}_{\mathrm{O} 2}$, including in the kidney [35]. Furthermore, chronic metformin treatment has been observed to decrease renal $\mathrm{Q}_{\mathrm{O} 2}$ and increase tissue $p \mathrm{O}_{2}$ [38]. Our results support this concept: we found that the healthy kidney relies on anaerobic metabolism upon metformin treatment, even with an increased oxygen supply. Our study has confirmed that acute metformin treatment alters cellular redox status in the kidneys, which has been proposed to be the result of inhibited mGPD [2].

The diabetic animals showed a markedly different response to the acute metformin treatment, as they were largely metabolically unaffected by the metformin treatment, evidenced by maintained PDH flux (bicarbonate/pyruvate ratio) and LDH flux (lactate/pyruvate ratio). We can conclude that metformin increases intrarenal lactate production acutely due to an altered redox capacity. This alteration is attenuated by the increased basal lactate production and an already elevated redox state in the kidney tissue from the diabetic animals.

Interestingly, attempts to change the cytosolic and mitochondrial redox potential have been reported to protect organ function in several disease states, including diabetes [39]. In the kidney in diabetes, a decreased $\mathrm{NAD}^{+} / \mathrm{NADH}$ ratio due to an accelerated polyol pathway has been demonstrated to blunt diabetic complications by normalising the redox state $\left(\mathrm{NAD}^{+} /\right.$ NADH ratio) [20, 23, 40].

Control animals receiving only saline injection show no detectable metabolic alterations following the infusion of the 
two supraphysiological ( $125 \mathrm{mmol} / \mathrm{l}$, substrate saturated condition) $\left[1-{ }^{13} \mathrm{C}\right]$ pyruvate infusions within the time frame of the experiment. This is in agreement with previous reports in the literature showing a minor influence on the metabolic pattern with sequential measurements, with a minimum of $15 \mathrm{~min}$ between infusions in rodents and $30 \mathrm{~min}$ in large animals [20, 41-43]. Moreover, metformin has been proposed to have anticancer effects by activating the AMPK pathway, and our result showing increased p-AMPK further supports that metformin can activate the AMPK pathway.

These novel findings indicate that the effect of metformin is predominantly due to the redox change, which highlights the possibility of targeting the altered redox balance in order to protect kidney function.

Acknowledgements The technical support of H. Vestergaard Nielsen (MR Research Centre, Department of Clinical Medicine, Aarhus University) and A. Fasching (Department of Medical Cell Biology, Uppsala University) are gratefully acknowledged.

Data availability Data is available on request. Correspondence and requests for materials should be addressed to C Lautsen (cl@ clin.au.dk).

Funding The study was supported by the Sundhed og Sygdom, Det Frie Forskningsråd, Aarhus University research fund.

Duality of interest The authors declare that there is no duality of interest associated with this manuscript.

Contribution statement HQ wrote the manuscript and contributed to acquisition and analysis of data. PMN and LBB contributed to acquisition of data and revised the manuscript. MS contributed to the acquisition of data, revised the manuscript and contributed to discussion. FP and CL conceived and designed the study, contributed to the analysis and interpretation of data, contributed to discussion, and wrote and reviewed the manuscript. All authors approved the final version to be published. CL is the guarantor of this work and, as such, had full access to all the data in the study and takes responsibility for the integrity of the data and the accuracy of the data analysis.

\section{References}

1. Hur KY, Lee MS (2015) New mechanisms of metformin action: focusing on mitochondria and the gut. J Diabetes Investig 6:600609

2. Madiraju AK, Erion DM, Rahimi Y et al (2014) Metformin suppresses gluconeogenesis by inhibiting mitochondrial glycerophosphate dehydrogenase. Nature 510:542-546

3. Vella S, Buetow L, Royle P, Livingstone S, Colhoun HM, Petrie JR (2010) The use of metformin in type 1 diabetes: a systematic review of efficacy. Diabetologia 53:809-820

4. Inzucchi SE, Maggs DG, Spollett GR et al (1998) Efficacy and metabolic effects of metformin and troglitazone in type II diabetes mellitus. N Engl J Med 338:867-872

5. Hundal RS, Krssak M, Dufour S et al (2000) Mechanism by which metformin reduces glucose production in type 2 diabetes. Diabetes 49:2063-2069
6. Frandsen CS, Dejgaard TF, Madsbad S (2016) Non-insulin drugs to treat hyperglycaemia in type 1 diabetes mellitus. Lancet Diabetes Endocrinol 4:766-780

7. Libman IM, Miller KM, DiMeglio LA et al (2015) Effect of metformin added to insulin on glycemic control among overweight/ obese adolescents with type 1 diabetes: a randomized clinical trial. JAMA 314:2241-2250

8. Petrie JR, Chaturvedi N, Ford I et al (2017) Metformin in adults with type 1 diabetes: design and methods of reducing with metformin vascular adverse lesions (REMOVAL): an international multicentre trial. Diabetes Obes Metab 19:509-516

9. Capitanio S, Marini C, Sambuceti G, Morbelli S (2015) Metformin and cancer: technical and clinical implications for FDG-PET imaging. World J Radiol 7:57-60

10. Cazzaniga M, Bonanni B (2015) Relationship between metabolic reprogramming and mitochondrial activity in cancer cells. Understanding the anticancer effect of metformin and its clinical implications. Anticancer Res 35:5789-5796

11. Mayer MJ, Klotz LH, Venkateswaran V (2015) Metformin and prostate cancer stem cells: a novel therapeutic target. Prostate Cancer Prostatic Dis 18:303-309

12. Pizzuti L, Vici P, Di Lauro L et al (2015) Metformin and breast cancer: basic knowledge in clinical context. Cancer Treat Rev 41: 441-447

13. Pollak $\mathrm{MN}$ (2012) Investigating metformin for cancer prevention and treatment: the end of the beginning. Cancer Discov 2:778-790

14. Chae YK, Arya A, Malecek MK et al (2016) Repurposing metformin for cancer treatment: current clinical studies. Oncotarget 7: 40767-40780

15. Nye HJ, Herrington WG (2011) Metformin: the safest hypoglycaemic agent in chronic kidney disease? Nephron Clini Pract 118:c380-c383

16. Inzucchi SE, Lipska KJ, Mayo H, Bailey CJ, McGuire DK (2014) Metformin in patients with type 2 diabetes and kidney disease: a systematic review. JAMA 312:2668-2675

17. Lewis AJ, Miller JJ, McCallum C et al (2016) Assessment of metformin-induced changes in cardiac and hepatic redox state using hyperpolarized $\left[1-{ }^{13} \mathrm{C}\right]$ pyruvate. Diabetes 65:3544-3551

18. Laustsen C, Ostergaard JA, Lauritzen MH et al (2013) Assessment of early diabetic renal changes with hyperpolarized $\left[1-{ }^{13}\right.$ C]pyruvate. Diabetes Metab Res Rev 29:125-129

19. von Morze C, Chang GY, Larson PE et al (2017) Detection of localized changes in the metabolism of hyperpolarized gluconeogenic precursors 13 C-lactate and $13 \mathrm{C}$-pyruvate in kidney and liver. Magn Reson Med 77:1429-1437

20. Laustsen C, Lycke S, Palm F et al (2014) High altitude may alter oxygen availability and renal metabolism in diabetics as measured by hyperpolarized [1-(13)C]pyruvate magnetic resonance imaging. Kidney Int 86:67-74

21. Kuo CC, Yeh HC, Chen B, Tsai CW, Lin YS, Huang CC (2015) Prevalence of metformin use and the associated risk of metabolic acidosis in US diabetic adults with CKD: a national cross-sectional study. Medicine 94:e2175

22. Eppenga WL, Lalmohamed A, Geerts AF et al (2014) Risk of lactic acidosis or elevated lactate concentrations in metformin users with renal impairment: a population-based cohort study. Diabetes Care 37:2218-2224

23. Runge $\mathrm{S}$, Mayerle J, Warnke $\mathrm{C}$ et al (2008) Metformin-associated lactic acidosis in patients with renal impairment solely due to drug accumulation? Diabetes Obes Metab 10:91-93

24. Persson P, Hansell P, Palm F (2012) NADPH oxidase inhibition reduces tubular sodium transport and improves kidney oxygenation in diabetes. Am J Physiol Regul Integr Comp Physiol 302:R1443R1449

25. Nordquist L, Brown R, Fasching A, Persson P, Palm F (2009) Proinsulin C-peptide reduces diabetes-induced glomerular 
hyperfiltration via efferent arteriole dilation and inhibition of tubular sodium reabsorption. Am J Physiol Ren Physiol 297:F1265F1272

26. Ardenkjaer-Larsen JH, Fridlund B, Gram A et al (2003) Increase in signal-to-noise ratio of $>10,000$ times in liquid-state NMR. Proc Natl Acad Sci U S A 100:10158-10163

27. Hurd RE, Yen YF, Chen A, Ardenkjaer-Larsen JH (2012) Hyperpolarized ${ }^{13} \mathrm{C}$ metabolic imaging using dissolution dynamic nuclear polarization. J Magn Reson Imaging 36:1314-1328

28. Laustsen C (2016) Hyperpolarized renal magnetic resonance imaging: potential and pitfalls. Front Physiol 7:72

29. Bertelsen LB, Nielsen PM, Qi H et al (2017) Diabetes induced renal urea transport alterations assessed with 3D hyperpolarized $13 \mathrm{C}, 15$ N-urea. Magn Reson Med 77:1650-1655

30. Qi H, Norlinger TS, Nielsen PM et al (2016) Early diabetic kidney maintains the corticomedullary urea and sodium gradient. Phys Rep 4:e12714. 10.14814/phy2.12714

31. Nielsen PM, Laustsen C, Bertelsen LB et al (2017) In situ lactate dehydrogenase activity: a novel renal cortical imaging biomarker of tubular injury? Am J Physiol Ren Physiol 312:F465-F473

32. Laustsen C, Nielsen PM, Norlinger TS et al (2017) Antioxidant treatment attenuates lactate production in diabetic nephropathy. Am J Physiol Ren Physiol 312:F192-F199

33. Owen MR, Doran E, Halestrap AP (2000) Evidence that metformin exerts its anti-diabetic effects through inhibition of complex 1 of the mitochondrial respiratory chain. Biochem $\mathrm{J}$ $348 \operatorname{Pt}(3): 607-614$

34. Baur JA, Birnbaum MJ (2014) Control of gluconeogenesis by metformin: does redox trump energy charge? Cell Metab 20:197-199
35. Protti A, Fortunato F, Monti M et al (2012) Metformin overdose, but not lactic acidosis per se, inhibits oxygen consumption in pigs. Crit Care 16:R75

36. Viollet B, Guigas B, Sanz Garcia N, Leclerc J, Foretz M, Andreelli F (2012) Cellular and molecular mechanisms of metformin: an overview. Clin Sci 122:253-270

37. Dobre M, Rahman M, Hostetter TH (2015) Current status of bicarbonate in CKD. J Am Soc Nephrol 26:515-523

38. Takiyama Y, Harumi T, Watanabe J et al (2011) Tubular injury in a rat model of type 2 diabetes is prevented by metformin: a possible role of HIF-1alpha expression and oxygen metabolism. Diabetes 60:981-992

39. Ying W (2008) NAD+/NADH and NADP+/NADPH in cellular functions and cell death: regulation and biological consequences. Antioxid Redox Signal 10:179-206

40. Tilton RG, Chang K, Nyengaard JR, Van den Enden M, Ido Y, Williamson JR (1995) Inhibition of sorbitol dehydrogenase. Effects on vascular and neural dysfunction in streptozocininduced diabetic rats. Diabetes 44:234-242

41. Laustsen C, Hansen ES, Kjaergaard U, Bertelsen LB, Ringgaard S, Stodkilde-Jorgensen H (2015) Acute porcine renal metabolic effect of endogastric soft drink administration assessed with hyperpolarized [1-(13)C]pyruvate. Magn Reson Med 74:558-563

42. Hansen ESS, Tougaard RS, Norlinger TS et al (2017) Imaging porcine cardiac substrate selection modulations by glucose, insulin and potassium intervention: a hyperpolarized $\left[1-{ }^{13} \mathrm{C}\right]$ pyruvate study. NMR Biomed 30:e3702

43. Atherton HJ, Schroeder MA, Dodd MS et al (2011) Validation of the in vivo assessment of pyruvate dehydrogenase activity using hyperpolarised ${ }^{13} \mathrm{C}$ MRS. NMR Biomed 24:201-208 